\title{
BioUML - universal platform for analyses of biomedical data
}

Fedor A. Kolpakov

Institute of Computational

Technologies SB RAS

BIOSOFT.RU, LLC

Novosibirsk, Russia

fedor@biosoft.ru

Anna S. Ryabova

Institute of Computational

Technologies SB RAS

BIOSOFT.RU, LLC

Novosibirsk, Russia

anna@biosoft.ru

Elena O. Kutumova

Institute of Computational

Technologies SB RAS

BIOSOFT.RU, LLC

Novosibirsk, Russia

elena.kutumova@biosoft.ru
Ivan S. Evshin

Institute of Computational

Technologies SB RAS

BIOSOFT.RU, LLC

Novosibirsk, Russia

ivan@biosoft.ru

Yury V. Kondrakhin

Institute of Computational

Technologies SB RAS

BIOSOFT.RU, LLC

Novosibirsk, Russia

yvkondrat@mail.ru

Nikita V. Mandrik

Institute of Computational

Technologies SB RAS

BIOSOFT.RU, LLC

Novosibirsk, Russia

manikitos@biosoft.ru
Ilya N. Kiselev

Institute of Computational

Technologies SB RAS

BIOSOFT.RU, LLC

Novosibirsk, Russia

axec@biosoft.ru

Sergey S. Pintus

Institute of Computational

Technologies SB RAS

BIOSOFT.RU, LLC

Novosibirsk, Russia

sspintus@biosoft.ru

Alexander E. Kel

BIOSOFT.RU, LLC

Novosibirsk, Russia

geneXplain $\mathrm{GmbH}$,

Wolfebuttel, Germany

alexander.kel@biosoft.ru

\begin{abstract}
BioUML (homepage: http://www.biouml.org, main public server: https://ict.biouml.org) is a web-based integrated environment (platform) for systems biology and analysis of biomedical data generated by omics technologies. Here we are presenting further extensions of BioUML platform that make it universal platform for analysis of wide range of biomedical data: 1) integration with Docker technology that allows easily pack and run existing software and databases; 2) integration of noVNC web client to work with software with rich graphic interface via web; 3) new integration with Galaxy and tool shed that allows reuse wide range of existing software for data analysis; 4) integration with Jupyter hub for interactive data analysis; 5) support of Common Workflow Language (CWL).
\end{abstract}

Keywords - BioUML, Docker, Galaxy, Jupyter notebooks, CWL, data analysis

\section{Introduction}

BioUML is a web-based integrated environment (platform) for systems biology and the analysis of biomedical data generated by omics technologies[1]. BioUML spans a comprehensive range of capabilities, including access to biological databases, powerful tools for systems biology (visual modelling, simulation, parameters fitting and analyses), a genome browser, scripting ( $R$, JavaScript) and a workflow engine. Due to integration with the Galaxy platform and R/Bioconductor, BioUML provides powerful possibilities for the analyses of omics data. The plug-in-based architecture allows the user to add new functionalities using plug-ins. To support collaborative work on scientific projects, there is a central authentication and authorization system (https://bio-store.org). The diagram editor enables several remote users to simultaneously edit diagrams.
Results

Figure 1 demonstrates the suggested architecture of universal platform for analysis of wide range of biomedical data:

1) Uniformly packed and described computer programs for data analysis and visualization:

- Docker technology is used to easily pack and run existing software and databases;

- Docker registry is used as repository packed software images;

- Common Workflow Language (CWL) and Galaxy formats are used to describe computer programs and parameters to start them.

2) Core - BioUML platform [1]. It also provides communication with integrated tools (Galaxy, Jupyter hub, $\mathrm{R}$, Docker, noVNC, etc.).

3) IT infrastructure where the platform was installed.

4) external resources can be used to store users data. They can be connected to the platform via specialised API. .

\section{ACKNOWLEDGMENT}

This study was supported by the Russian Science Foundation, grant No. 19-14-00295.

\section{REFERENCES}

[1] Kolpakov F, Akberdin I, Kashapov T, Kiselev L, Kolmykov S, Kondrakhin Y, Kutumova E, Mandrik N, Pintus S, Ryabova A, Sharipov R. BioUML: an integrated environment for systems biology and collaborative analysis of biomedical data. Nucleic acids research. 2019 Jul 2:47(W1):W225-33. 

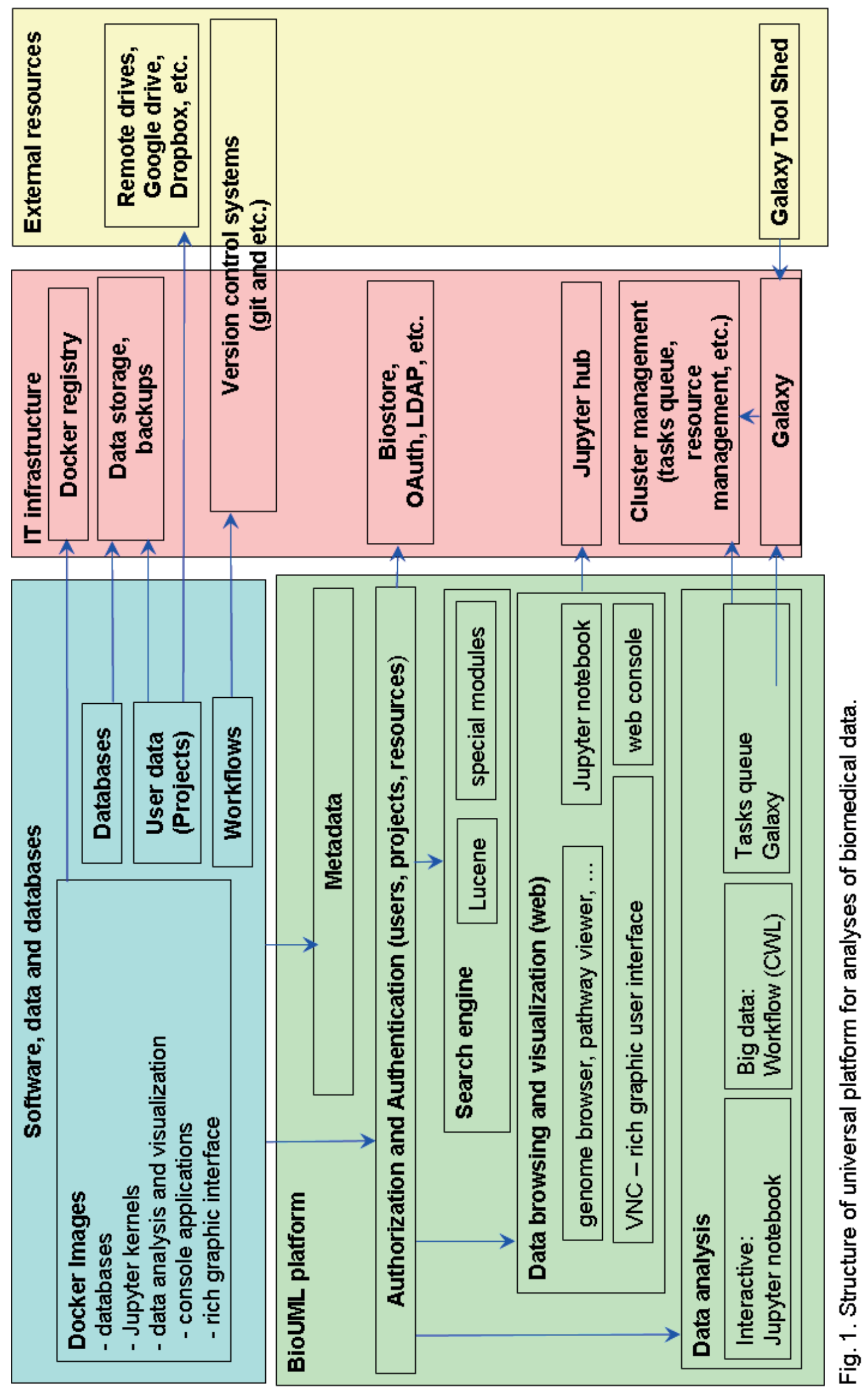